\title{
Puerarin reduces ischemia/reperfusion-induced myocardial injury in diabetic rats via upregulation of vascular endothelial growth factor A/angiotensin-1 and suppression of apoptosis
}

\author{
BAO-QIANG GUO ${ }^{1 *}$, JING-BO XU $^{2 *}$, MING XIAO $^{3,4^{*}}$, MIN DING $^{5}$ and LI-JUN DUAN ${ }^{6}$ \\ ${ }^{1}$ Department of Endocrinology, The Second People's Hospital of Liaocheng, Linqing, Shandong 252601; ${ }^{2}$ Department of \\ Endocrinology, Jiamusi Central Hospital, Jiamusi, Heilongjiang 154002; ${ }^{3}$ Department of Cardiology, Qilu Hospital, Shandong \\ University, Jinan, Shandong 250012; ${ }^{4}$ Department of Cardiology, Shandong Jiaotong Hospital, Jinan, Shandong 250031; \\ ${ }^{5}$ Department of Podiatry, 2011 Collaborative Innovation Center of Tianjin for Medical Epigenetics, Key Laboratory of Hormones \\ and Development (Ministry of Health), Metabolic Diseases Hospital and Tianjin Institute of Endocrinology, Tianjin Medical \\ University, Tianjin, Hebei 300070; ${ }^{6}$ Department of Endocrinology, Tianjin First Central Hospital, Tianjin, Hebei 300192, P.R. China
}

Received April 25, 2016; Accepted May 16, 2017

DOI: $10.3892 / \mathrm{mmr} .2018 .8754$

\begin{abstract}
Puerarin is an active ingredient of pueraria, which has been developed for puerarin injections, used in the treatment of cardiovascular diseases including arrhythmia, myocardial ischemia and hypertension. However, the molecular mechanisms of puerarin on ischemia/reperfusion (I/R)-induced myocardial apoptosis in diabetic rats are not fully understood. The present study aimed to investigate whether puerarin can attenuate I/R-induced myocardial apoptosis in diabetic rats, and to investigate the underlying mechanism. A hemodynamic analyzing system was employed to analyze the left ventricular developed pressure (LVDP), the left ventricular end-systolic interior dimension (LVIDs) and the left ventricular end diastolic interior dimension (LVIDd). ELISA kits were used to analyze malondialdehyde (MDA), superoxide dismutase (SOD), tumor necrosis factor- $\alpha$ (TNF- $\alpha$ ) and interleukin (IL)-6 levels, NO production and caspase-3 activity. Nuclear factor (NF)- $\mathrm{B}$, ascular endothelial growth factor A (VEGFA), angiotensin (Ang)-I, phosphorylated (p)-endothelial nitric oxide synthase protein expression was analyzed using western blot analysis. Puerarin significantly reduced the myocardial infarct area, and increased left ventricular developed pressure in diabetic rats with myocardial I/R. Oxidative stress, inflammation and nuclear factor- $\kappa \mathrm{B}$ protein expression were significantly reduced by puerarin. Furthermore, puerarin activated the
\end{abstract}

Correspondence to: Dr Li-Jun Duan, Department of Endocrinology, Tianjin First Central Hospital, 24 Fu Kang Road, Tianjin, Hebei 300192, P.R. China

E-mail: dun968719085@126.com

${ }^{*}$ Contributed equally

Key words: puerarin, ischemia/reperfusion-induced myocardial, vascular endothelial growth factor A, angiotensin-1 protein expression levels of VEGFA and Ang-I, and increased nitric oxide production, phosphorylated-endothelial nitric oxide synthase protein expression and caspase-3 activity. These results demonstrated that the myocardial protective effect of puerarin serves to reduce myocardial I/R injury, via upregulation of VEGFA/Ang-1 and suppression of apoptosis, in diabetic rats with myocardial $\mathrm{I} / \mathrm{R}$.

\section{Introduction}

Diabetes mellitus (DM) is a chronic disease that exhibits genetic susceptibility (1). DM is caused by decreased levels or an absolute lack of insulin, resulting from the interaction of various internal and external factors, and is characterized by high blood glucose and lipid metabolism of sugar (1). Diabetic patients are disposed to complications in early and late stages, including nephropathy, eye disease, foot pathologies and cardiomyopathy; diabetic cardiomyopathy belongs to the cardiovascular complications of diabetes and is the predominant cause of mortality in patients with diabetes (2).

Angiotensin-converting enzyme (ACE) 2 is the only homologue of ACE that has been identified to date; it exhibits $42 \%$ homologous sequences with ACE (3). ACE2 is an inner membrane carboxypeptidase that can hydrolyze angiotensin 1 (Ang-1) into Ang-(1-9), and this may be further hydrolyzed by ACE into Ang-(1-7) (4). ACE2 can also remove the terminal tryptophan of Ang-II, thus directly hydrolyzing Ang-II to Ang-(1-7). Furthermore, in vitro studies have demonstrated that the enzymatic activity of ACE2 on Ang-II is 400 times of that on Ang-I (4). The binding of Ang-(1-7) with its specific G-protein coupled receptor Mas promotes the release of bradykinin, nitric oxide (NO) and prostaglandins, which serve roles in vessel expansion, decreasing blood pressure, improving insulin resistance, resisting inflammation, inhibiting cell proliferation, anticoagulation and protecting the vascular endothelium (5). ACE2 hydrolyzes vasopressin, neurotensin and dynorphin A-(1-13), which are involved in cardiovascular 
regulation. Cardiovascular protection is enabled by maintaining homeostasis of the renin-angiotensin system, by modulating the antagonizing effect of the ACE2/Ang-(1-7)/Mas receptor axis on the ACE/Ang-1/Ang-II-type 1 (ATI) receptor axis (6).

The vascular endothelial growth factor (VEGF) family serves a major role in the regulation of the angiogenesis network. VEGF has direct effects on vascular endothelial cells by promoting their proliferation and migration; VEGF is also a critical regulator of angiogenesis in the formation human tumors (7). Furthermore, VEGF may be involved in regulating the interaction between endothelial cells and between endothelial cells and the basement membrane, by interacting with receptors on vascular endothelial cells (8).

Diabetic cardiomyopathy (DCM) is a chronic and complex pathological process that can alter cell metabolism, resulting in damage to the endoplasmic reticulum, mitochondria and other organelles, and ultimately result in myocardial hypertrophy and increased apoptosis (9). Furthermore, apoptosis resulting from altered energy metabolism of the cell serves an additional role in the pathology of DCM; large scale apoptosis can aggravate myocardial remodeling and cause further tissue dysfunction, thus creating a destructive pathogenic cycle $(10,11)$.

The active ingredients of pueraria constitute isoflavones, including daidzin, daidzein and puerarin (12). Puerarin has wide pharmacological effects (12); aside from its application in the treatment of cardiovascular and cerebrovascular diseases, with broad prospects for development and clinical application (13). Puerarin inhibits lipid peroxidation and aldose reductase activity, removes superoxide ion radicals, and has a protective effect on endothelial cells (14). Furthermore, puerarin may significantly slow the glucosamine metabolism of endothelial cells, reduce endothelin and platelet surface activity, inhibit platelet aggregation and adhesion, lower blood lipids, cholesterol, blood viscosity and prevent thrombosis (14). Therefore, puerarin may be useful in the protection of vascular endothelial cells, the promotion of angiomalacia and the inhibition of atherosclerosis (15). The present study investigated whether the myocardial protective effects of puerarin can attenuate ischemia/reperfusion (I/R)-induced myocardial apoptosis in diabetic rats, via upregulation of VEGFA/Ang-1 and suppression of apoptosis in rats, and thus exert cardioprotective effects in diabetics.

\section{Materials and methods}

Animals and experimental groups. Male Sprague-Dawley rats (8-10 weeks old, $\mathrm{n}=40$ ) weighing 200-220 g were housed at $22-23^{\circ} \mathrm{C}, 55-60 \%$ humidity, $12 \mathrm{~h}$ light/dark cycle and access free to food and water, and randomly assigned into five groups: Sham ( $n=6)$; ischemia/reperfusion $(I / R ; n=10) ; I / R+L$ (lower dosage, $\mathrm{n}=8,25 \mathrm{mg} / \mathrm{kg}$ puerarin); I/R+M (medium dosage, $\mathrm{n}=8,50 \mathrm{mg} / \mathrm{kg}$ puerarin), I/R+H (higher dosage, $\mathrm{n}=8$, $100 \mathrm{mg} / \mathrm{kg}$ puerarin). Rats were injected intraperitoneally with streptozotocin $(30 \mathrm{mg} / \mathrm{kg})$ twice, with a rest day between each injection. The following week, the myocardial I/R model was induced with $35 \mathrm{mg} / \mathrm{kg}$ pentobarbital sodium; the heart was exteriorized with a left thoracic incision, and a slipknot made using 4-0 silk was placed around the left anterior descending coronary artery. Ischemia was performed for $30 \mathrm{~min}$, the slipknot was released and reperfusion was allowed to occur for $3 \mathrm{~h}$. Rats of the sham group were anesthetized with $35 \mathrm{mg} / \mathrm{kg}$ pentobarbital sodium and underwent a sham surgery in which the heart was exteriorized without myocardial I/R. The following day, myocardial I/R rats were gavaged with 25, 50 or $100 \mathrm{mg} / \mathrm{kg} /$ puerarin (Sigma-Aldrich; Merck KGaA, Darmstadt, Germany) every 2 days over 4 weeks. The present study was approved by the ethics committee of Tianjin First Central Hospital (Tianjin, China).

Assessments of myocardial function. After treatment with puerarin for 4 weeks, A hemodynamic analyzing system (Chengdu Xinjin Shifeng Medical Apparatus \& Instrument Co., Ltd., Chengdu, China) was employed to analyze the left ventricular developed pressure (LVDP), the left ventricular end-systolic interior dimension (LVIDs) and the left ventricular end diastolic interior dimension (LVIDd) as described in a recent study (16). Under anesthesia (35 $\mathrm{mg} / \mathrm{kg}$ of pentobarbital sodium), the coronary artery was ligated; after $4 \mathrm{~h}$, the left ventricle was stored at $-80^{\circ} \mathrm{C}$ for $30 \mathrm{~min}$. The left ventricle was subsequently sliced into 4-mm thick sections to assess the size of the infarct. The heart area assessed was the ischemic heart muscles. The infarct size area was assessed by volume and weight as a percentage of the left ventricle.

Determination of malondialdehyde (MDA), superoxide dismutase (SOD), tumor necrosis factor- $\alpha(T N F-\alpha)$ and interleukin (IL)-6 levels, NO production and caspase-3 activity. Rats were anesthetized and blood was collected from the eye socket of each rat. Plasma was centrifuged at 8,000 x $\mathrm{g}$ for $10 \mathrm{~min}$ at $4^{\circ} \mathrm{C}$. ELISA kits obtained from Wuhan Elabscience Biotechnology Co., Ltd., (Wuhan, China) were employed to analyze the levels of MDA (E-EL-0060c), SOD(E-EL-R1424c), TNF- $\alpha$ (E-EL-R0019c) and IL-6 (E-EL-R0015c). Total NO production was measured using an NO analyzer (Nanjing Jiancheng Bioengineering Institute, Nanjing, China). Caspase-3 activity was measured using a Caspase 3 Activity Assay kit (Beyotime Institute of Biotechnology, Haimen, China). Absorbance values were measured using a Synergy HT microplate reader (BioTek Instruments, Inc., Winooski, VT, USA).

Western blot analysis. Heart tissue samples were lysed in radioimmunoprecipitation assay buffer (Beyotime Institute of Biotechnology) on ice for $30 \mathrm{~min}$, and the supernatant was centrifuged at $8,000 \mathrm{x}$ g for $10 \mathrm{~min}$ at $4^{\circ} \mathrm{C}$. Following quantification of the protein concentration using a Bicinchoninic Acid Protein Assay kit (Thermo Fisher Scientific, Inc., Waltham, MA, USA), proteins $(50 \mu \mathrm{g})$ were separated by $10 \%$ SDS-PAGE and transferred onto Immobilon-FL transfer membranes (EMD Millipore, Billerica, MA, USA). Membranes were blocked with 5\% non-fat milk for $1 \mathrm{~h}$ at room temperature and incubated overnight at $4^{\circ} \mathrm{C}$ with anti-nuclear factor (NF)- $\mathrm{B}$ (sc-71675, 1:1,000), anti-VEGFA (sc-7269, 1:1,000), anti-Ang-1 (cat. no. sc-6320, 1:1,000), anti-phosphorylated (p)-endothelial nitric oxide synthase (eNOS; sc-293032 1:1,000; all from Santa Cruz Biotechnology, Inc., Dallas, TX, USA) and anti-GAPDH (BM3876, 1:5,000; Wuhan Boster Biological Technology, Ltd., Wuhan, China). Blots were subsequently washed 3 times with TBS with 
$0.1 \%$ Tween-20 (TBST), and incubated with goat anti-mouse immunoglobulin G-horseradish peroxidase secondary antibodies (sc-2005, 1:5,000; Santa Cruz Biotechnology) in TBST solution for $1 \mathrm{~h}$ at $37^{\circ} \mathrm{C}$. Bands were visualized using BeyoECL Star (P0018A; Beyotime Institute of Biotechnology) quantified using the Quantity image analyzer 3.0 (Bio-Rad Laboratories, Inc., Hercules, CA, USA).

Statistical analysis. Data are expressed as the mean \pm standard error ( $n=3$ ) using SPSS 17.0 (SPSS, Inc. Chicago, IL, USA). Between-group differences were determined using one-way analysis of variance followed by Tukey's post-hoc analysis. $\mathrm{P}<0.05$ was considered to indicate a statistically significant difference.

\section{Results}

Puerarin reduces the myocardial infarct area in diabetic myocardial $I / R$ rats. The chemical structure of puerarin is presented in Fig. 1. The myocardial infarct area was markedly increased in the diabetic I/R model rats, compared with the sham control group. Treatment with puerarin for 4 weeks markedly reduced the myocardial infarct area, compared with untreated I/R diabetic rats (Fig. 2).

Puerarin increases the LVDP in diabetic myocardial I/R rats. A significantly attenuated LVDP in the diabetic I/R rat model group was observed, compared with the sham control group. Puerarin treatment increased the LVDP in diabetic myocardial I/R rats, compared with the diabetic I/R rat model group (Fig. 3A). Notably, a significant increase was observed in LVIDs and LVIDd in the diabetic I/R rat model group, compared with the control group (Fig. 3B and C). However, puerarin treatment markedly decreased LVIDs and LVIDd in diabetic I/R rats, compared with rats in the diabetic I/R model group.

Puerarin inhibits oxidative stress in diabetic myocardial I/R rats. MDA and SOD activity were measured to investigate the protective effect of puerarin on myocardial I/R in diabetic rats. Increased MDA activity (Fig. 4A) and decreased SOD activity (Fig. 4B) were observed in the diabetic myocardial I/R rat model, compared with the sham control group. Puerarin treatment reversed these alterations to MDA and SOD activity, compared with rats in the diabetic myocardial I/R model group (Fig. 4).

Puerarin inhibits inflammation in diabetic myocardial $I / R$ rats. The protective effects of puerarin on myocardial $\mathrm{I} / \mathrm{R}$ in diabetic rats were further examined by investigating the plasma levels of TNF- $\alpha$ and IL-6. TNF- $\alpha$ and IL- 6 levels were significantly increased in the plasma of rats in the diabetic myocardial I/R model, compared with the sham control group (Fig. 5A and B, respectively). However, treatment with puerarin significantly reduced TNF- $\alpha$ and IL- 6 levels, compared with rats in the diabetic myocardial I/R model group.

Puerarin inhibits $N F-\kappa B$ and VEGFA protein expression in diabetic myocardial $I / R$ rats. $\mathrm{NF}-\kappa \mathrm{B}$ and VEGFA protein expression levels were measured to examined the<smiles>O=c1c(-c2ccc(O)cc2)coc2c([C@@H]3O[C@H](CO)[C@@H](O)[C@H](O)[C@H]3O)c(O)ccc12</smiles>

Figure 1. Chemical structure of puerarin.

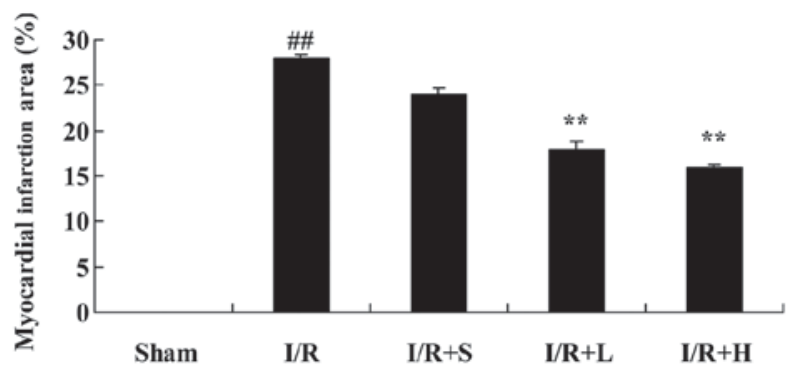

Figure 2. Puerarin reduces the myocardial infarct area in diabetic rats. Data are expressed as the mean \pm standard error. ${ }^{\# \#} \mathrm{P}<0.01$ vs. sham group, ${ }^{* *} \mathrm{P}<0.01$ vs. myocardial I/R group. Sham, sham group; I/R, myocardial I/R group; $\mathrm{I} / \mathrm{R}+\mathrm{L}$, myocardial $\mathrm{I} / \mathrm{R}+25 \mathrm{mg} / \mathrm{kg}$ puerarin group; $\mathrm{I} / \mathrm{R}+\mathrm{M}$, myocardial $\mathrm{I} / \mathrm{R}+50 \mathrm{mg} / \mathrm{kg}$ puerarin group; $\mathrm{I} / \mathrm{R}+\mathrm{H}$, myocardial $\mathrm{I} / \mathrm{R}+100 \mathrm{mg} / \mathrm{kg}$ puerarin group. I/R, ischemia/reperfusion injury.
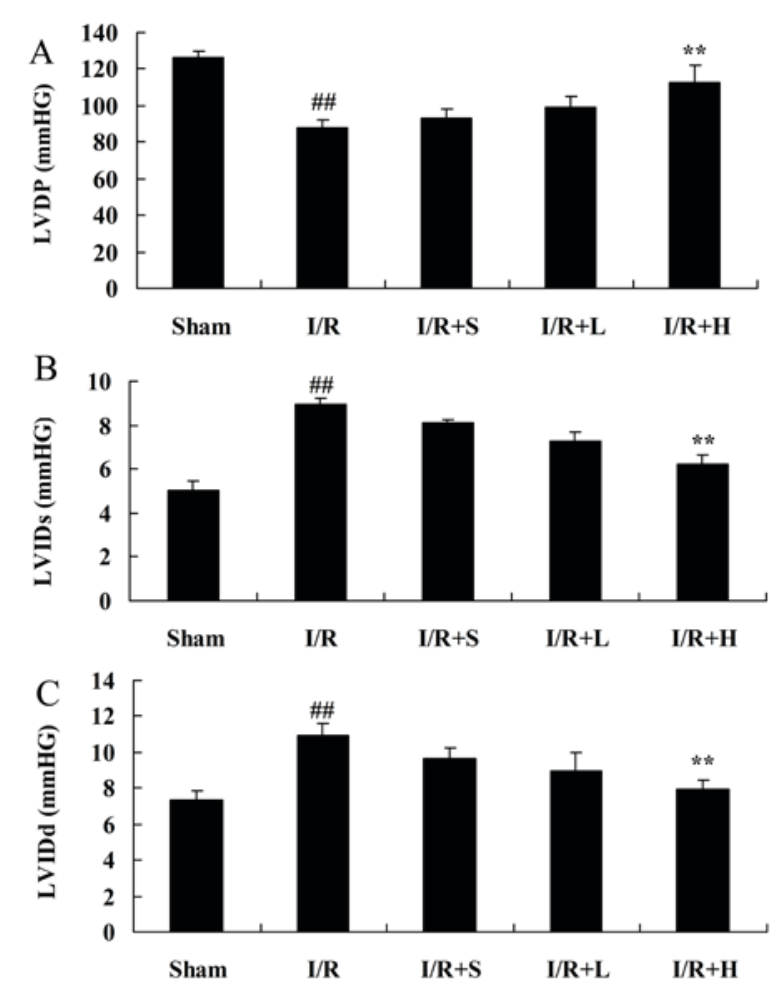

Figure 3. Puerarin increases the LVDP in diabetic myocardial I/R rats Puerarin increases (A) LVDP and decreases (B) LVIDs and (C) LVIDd. Data are expressed as the mean \pm standard error. ${ }^{\# \#} \mathrm{P}<0.01$ vs. sham group, ${ }^{* *} \mathrm{P}<0.01$ vs. myocardial I/R group. I/R, ischemia/reperfusion injury; Sham, sham group; I/R, myocardial I/R group; I/R+L, myocardial I/R $+25 \mathrm{mg} / \mathrm{kg}$ puerarin group; I/R+M, myocardial I/R $+50 \mathrm{mg} / \mathrm{kg}$ puerarin group; $\mathrm{I} / \mathrm{R}+\mathrm{H}$, myocardial I/R $+100 \mathrm{mg} / \mathrm{kg}$ puerarin group. I/R, ischemia/reperfusion injury. 

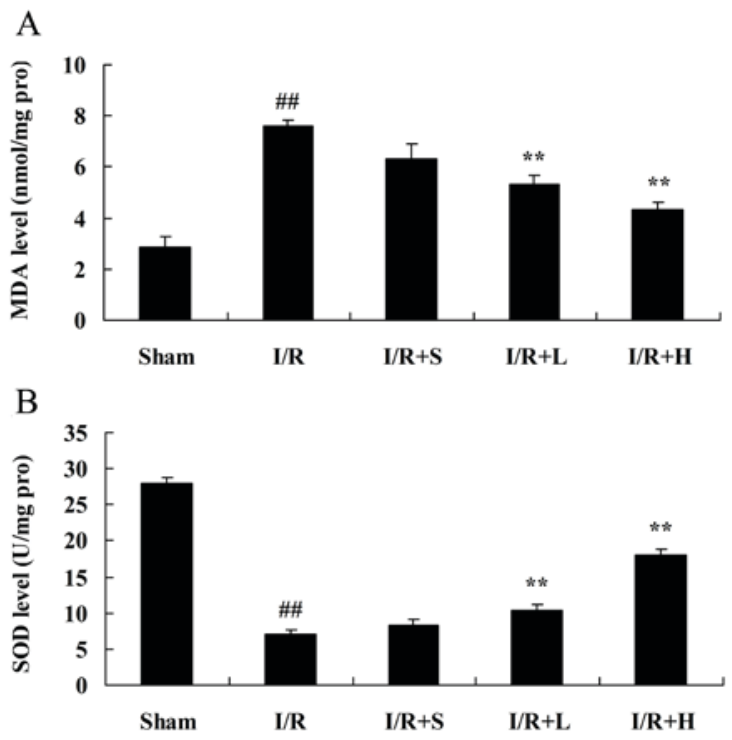

Figure 4. Puerarin inhibits oxidative stress in diabetic rats. Puerarin inhibits (A) MDA and (B) SOD activity in diabetic myocardial I/R rats. Data are expressed as the mean \pm standard error. ${ }^{\# \#} \mathrm{P}<0.01$ vs. sham group, ${ }^{* *} \mathrm{P}<0.01$ vs. myocardial I/R group. I/R, ischemia/reperfusion injury; Sham, sham group; I/R, myocardial I/R group; I/R+L, myocardial I/R $+25 \mathrm{mg} / \mathrm{kg}$ puerarin group; $\mathrm{I} / \mathrm{R}+\mathrm{M}$, myocardial $\mathrm{I} / \mathrm{R}+50 \mathrm{mg} / \mathrm{kg}$ puerarin group; $\mathrm{I} / \mathrm{R}+\mathrm{H}$, myocardial $\mathrm{I} / \mathrm{R}+100 \mathrm{mg} / \mathrm{kg}$ puerarin group. I/R, ischemia/reperfusion injury.
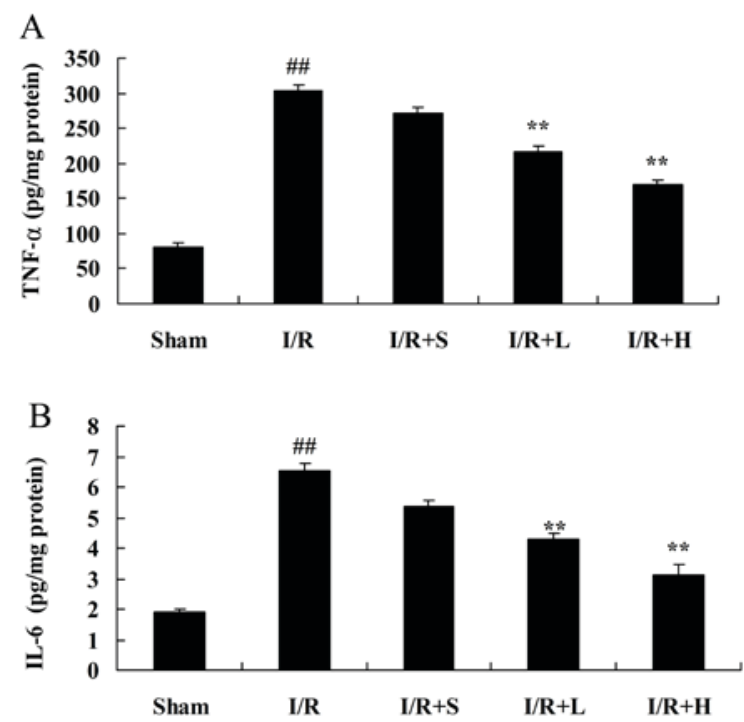

Figure 5. Puerarin reduces inflammation in diabetic myocardial I/R rats Puerarin inhibits (A) TNF- $\alpha$ and (B) IL-6 levels. Data are expressed as the mean \pm standard error. ${ }^{\# \#} \mathrm{P}<0.01$ vs. sham group, ${ }^{* *} \mathrm{P}<0.01$ vs. myocardial I/R group. I/R, ischemia/reperfusion injury; Sham, sham group; I/R, myocardial I/R group; I/R+L, myocardial I/R $+25 \mathrm{mg} / \mathrm{kg}$ puerarin group; $\mathrm{I} / \mathrm{R}+\mathrm{M}$, myocardial $\mathrm{I} / \mathrm{R}+50 \mathrm{mg} / \mathrm{kg}$ puerarin group; $\mathrm{I} / \mathrm{R}+\mathrm{H}$, myocardial $\mathrm{I} / \mathrm{R}+100 \mathrm{mg} / \mathrm{kg}$ puerarin group. I/R, ischemia/reperfusion injury.

anti-inflammatory effect of puerarin on diabetic myocardial $\mathrm{I} / \mathrm{R}$ in rats. NF- $\mathrm{\kappa B}$ protein expression was significantly induced and VEGFA protein expression was significantly suppressed in diabetic myocardial $\mathrm{I} / \mathrm{R}$ model rats, compared with the sham control group (Fig. 6). However, puerarin treatment significantly suppressed NF- $\mathrm{kB}$ and elevated VEGFA protein expression in puerarin-treated diabetic I/R rats, compared with the untreated diabetic $\mathrm{I} / \mathrm{R}$ model rats.
Puerarin increases Ang-1 and p-eNOS protein expression and $N O$ production in diabetic myocardial I/R rats. The role of Ang-1 and p-eNOS in puerarin-induced diabetic myocardial $\mathrm{I} / \mathrm{R}$ protection was investigated. Inhibition of Ang-1 and p-eNOS protein expression and NO production was observed in diabetic myocardial $\mathrm{I} / \mathrm{R}$ rats, compared with the sham control group (Fig. 7). Puerarin treatment significantly alleviated the I/R-induced inhibition of Ang-1 and p-eNOS protein expression and NO production, compared with the untreated diabetic myocardial I/R rats.

Puerarin decreases caspase-3 activity in diabetic myocardial $I / R$ rats. To investigate whether puerarin protects against apoptosis, caspase-3 activity was assessed (Fig. 8). Increased activation of caspase- 3 was observed in diabetic myocardial I/R rats, compared with the sham control group. Treatment with puerarin significantly decreased caspase-3 activity in untreated diabetic myocardial I/R rats.

\section{Discussion}

DCM is a chronic cardiac complication directly caused by diabetes, and is independent of coronary heart disease, hypertension and valvular heart disease $(17,18)$. Early stage DCM predominantly manifests with left ventricular hypertrophy and diastolic dysfunction (19). The left ventricular ejection score at this stage may be normal or even elevated; however, systolic dysfunction and a decline in the ejection fraction value are presented as the disease progresses, and these may ultimately result in heart failure (20). DCM pathology also includes cardiac hypertrophy and apoptosis, heart wall thickening, capillary basement membrane thickening, capillary endothelial lesions and microvascular lesions (10). The pathogenesis of DCM is complex; high blood sugar has been recognized as a leading risk factor, accompanied by lipid toxicity (triglycerides in the blood), oxidative stress, inflammation, autonomic neuropathy, microvascular disease and activation of renin-angiotensin-aldosterone system (20). In addition, mitochondrial dysfunction and epigenetic changes have been demonstrated to participate in the occurrence of DCM (21). The results of the present study indicated that puerarin may reduce the myocardial infarct area, increase LVDP and decrease LVIDs and LVIDd in diabetic myocardial $\mathrm{I} / \mathrm{R}$ rats.

High blood sugar and abnormal glucose metabolism induce excessive reactive oxygen species (ROS) production by the mitochondrial electron transportation chain. ROS and oxidative stress can cause DNA damage, meanwhile activating a DNA repair enzyme, poly ADP-ribose polymerase (22). Advanced glycation endproducts can activate nuclear factor kB (NF- $\mathrm{kB})$ via binding to the galectin- 3 receptor (23). The NF- $\mathrm{KB}$ pathway can regulate the expression of inflammation-related genes to increase the synthesis of TNF- $\alpha$ and interleukin, amongst others, resulting in myocardial damage (23). The present study discovered that puerarin may reduce oxidative stress and the expression of inflammatory cytokines and NF- $\mathrm{kB}$, in diabetic myocardial I/R rats. Furthermore, Li et al (12) demonstrated that puerarin can reduce diabetic aorta injury via the suppression of NADPH oxidase-induced oxidative stress and NF- $\kappa B$ p65 in diabetic rats. 
A

B

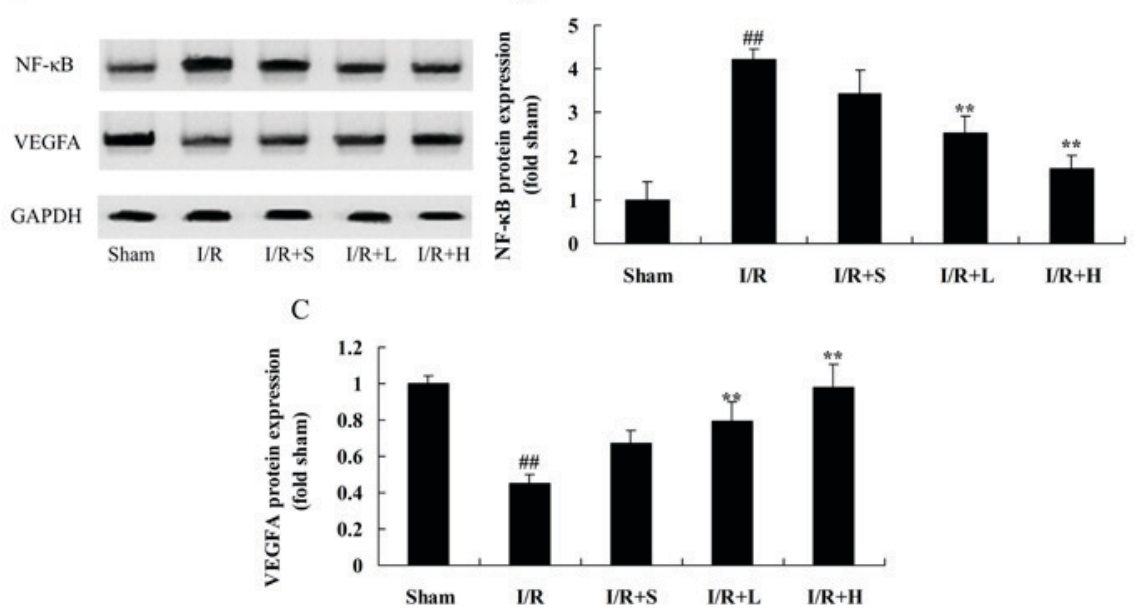

Figure 6. Puerarin inhibits NF- $\mathrm{kB}$ and increases VEGFA protein expression in diabetic myocardial I/R rats. (A) Western blot analysis indicated that puerarin inhibits NF- $\mathrm{kB}$ and increases VEGFA protein expression. Quantification of (B) NF- $\mathrm{kB}$ and (C) VEGFA protein expression. Data are expressed as the mean \pm standard error. ${ }^{\# /} \mathrm{P}<0.01$ vs. sham group, ${ }^{* *} \mathrm{P}<0.01$ vs. myocardial I/R group. I/R, ischemia/reperfusion injury; Sham, sham group; I/R, myocardial I/R group; I/R+L, myocardial I/R $+25 \mathrm{mg} / \mathrm{kg}$ puerarin group; $/ \mathrm{R}+\mathrm{M}$, myocardial I/R $+50 \mathrm{mg} / \mathrm{kg}$ puerarin group; $\mathrm{I} / \mathrm{R}+\mathrm{H}, \mathrm{myocardial} \mathrm{I} / \mathrm{R}+100 \mathrm{mg} / \mathrm{kg}$ puerarin group. I/R, ischemia/reperfusion injury.

A

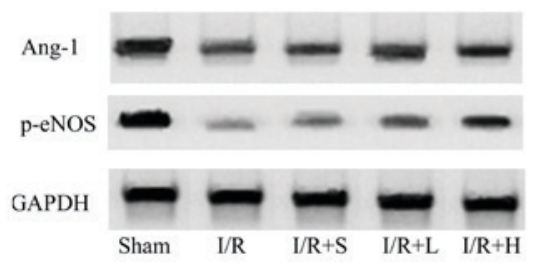

$\mathrm{C}$

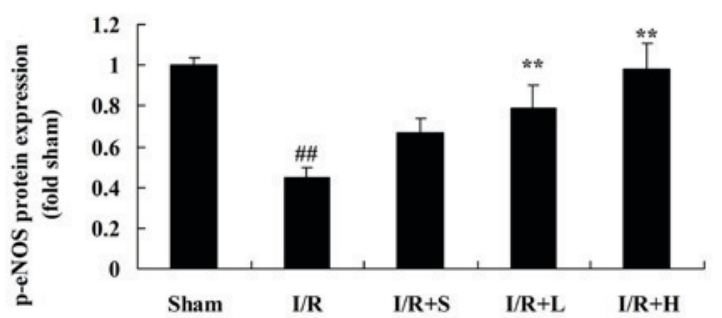

B

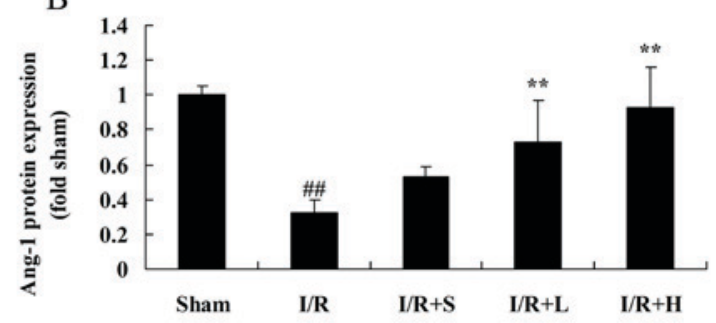

$\mathrm{D}$

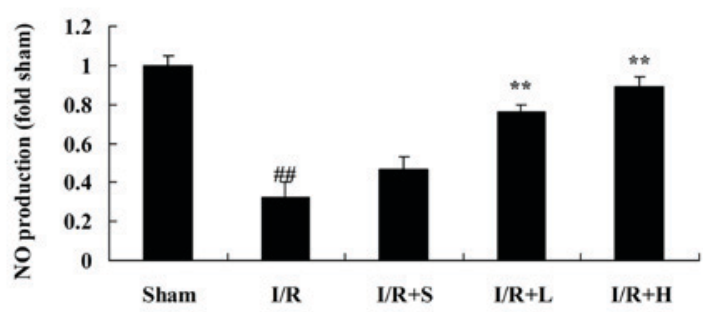

Figure 7. Puerarin increases Ang-1 and p-eNOS protein expression in diabetic myocardial I/R rats. (A) Western blot analysis indicated that puerarin inhibits Ang-1 and p-eNOS protein expression. Quantification of (B) Ang-1 and (C) p-eNOS protein expression. (D) Analysis of NO production. ${ }^{\# \# P}<0.01$ vs. sham group, ${ }^{* *} \mathrm{P}<0.01$ vs. myocardial I/R group. I/R, ischemia/reperfusion injury; Sham, sham group; I/R, myocardial I/R group; I/R+L, myocardial I/R + $25 \mathrm{mg} / \mathrm{kg}$ puerarin group; I/R+M, myocardial I/R $+50 \mathrm{mg} / \mathrm{kg}$ puerarin group; $\mathrm{I} / \mathrm{R}+\mathrm{H}$, myocardial $\mathrm{I} / \mathrm{R}+100 \mathrm{mg} / \mathrm{kg}$ puerarin group. I/R, ischemia/reperfusion injury.

Ang-(1-7) predominantly associates with the Mas receptor; however, a low amount of Ang-(1-7) also binds with the Ang-II type 2 (AT2) receptor (6). Association of Ang-(1-7) with the AT2 receptor can antagonize the ATI receptor; activation of eNOS promotes the release of NO, prostacyclin and other vasodilators; this increases the activity of bradykinin and thus antagonizes the effect of Ang-II (5). Furthermore, association of Ang-(1-7) with the Mas receptor can counteract the induction of vasoconstriction induced by Ang-II binding to the ATI receptor (24). In addition, VEGFA is a potent wound healing cytokine; the main functions of which include inducing angiogenesis, promoting endothelial cell proliferation and enhancing microvascular permeability, which results in widespread leakage of plasma proteins (25).

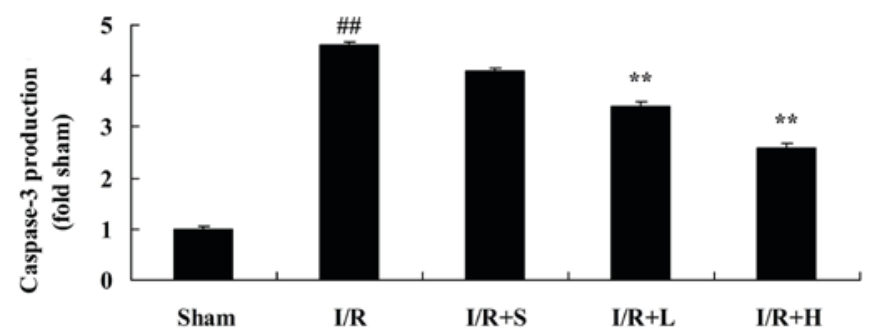

Figure 8. Puerarin reduces caspase-3 activity in diabetic myocardial I/R rats. Data are expressed as the mean \pm standard error. ${ }^{\# \#} \mathrm{P}<0.01$ vs. sham group, ${ }^{* *} \mathrm{P}<0.01$ vs. myocardial I/R group. I/R, ischemia/reperfusion injury; Sham, sham group; I/R, myocardial I/R group; I/R+L, myocardial I/R $+25 \mathrm{mg} / \mathrm{kg}$ puerarin group; $\mathrm{I} / \mathrm{R}+\mathrm{M}$, myocardial $\mathrm{I} / \mathrm{R}+50 \mathrm{mg} / \mathrm{kg}$ puerarin group; $\mathrm{I} / \mathrm{R}+\mathrm{H}$, myocardial I/R $+100 \mathrm{mg} / \mathrm{kg}$ puerarin group. I/R, ischemia/reperfusion injury. 
These proteins directly or indirectly alter the extracellular matrix components to form a temporary new matrix; this matrix supports the migration of endothelial cells and fibroblasts, which is conducive to wound repair. The present study demonstrated that puerarin significantly increases VEGFA, Ang-1 and p-eNOS protein expression, and activates NO production in diabetic myocardial I/R rats. Ai et al (15) demonstrated that puerarin accelerates cardiac angiogenesis and improves cardiac function via upregulation of VEGFA, Ang-1 and Ang-2.

Proliferation and apoptosis occur in the early stages of DCM cardiomyocyte hypertrophy; however, normal heart function can be maintained (26). As the disease develops, the myocardial intracellular environment becomes disordered; myocardial cells lose normal regulation and the rate of apoptosis exceeds the speed of cell proliferation; the apoptotic area increases, and the resulting loss of large numbers of cells eventually leads to a significant reduction in cardiac function $(27,28)$. The regulation of apoptosis involves a series of complex cascades, the most important of which are the caspase-related apoptosis signal transduction pathways, which include the death receptor-mediated apoptosis signaling pathway, the mitochondrial/cytochrome $c$-mediated apoptosis pathway and the endoplasmic reticulum stress-mediated apoptosis pathway (29). In the present study, treatment with puerarin significantly decreased caspase- 3 activity in diabetic rats. Notably, Iribarren et al (25) demonstrated that puerarin may protect against oxidative stress injury via the downregulation of caspase- 3 in neural cells.

In conclusion, the results of the present study indicated that puerarin markedly reduces the myocardial infarct area, increases LVDP and decreases LVIDs and LVIDd in diabetic myocardial I/R rats. Furthermore, puerarin may reduce oxidative stress and the expression of inflammatory cytokines via the inhibition of NF- $\mathrm{KB}$, upregulation of the VEGFA/Ang-1 signaling pathway and the suppression of apoptosis in diabetic rats. The results of the present study indicated that puerarin may be useful as a myocardial protective treatment to reduce cardiomyopathy in diabetic patients.

\section{Acknowledgements}

The present study was supported by the Health Bureau of Science and Technology fund of Tianjin (grant no. 2015KZ092).

\section{Competing interests}

The authors declare that they have no competing interests.

\section{References}

1. Lingvay I, Pérez Manghi F, García-Hernández P, Norwood P, Lehmann L, Tarp-Johansen MJ and Buse JB; DUAL V Investigators: Effect of insulin glargine up-titration vs insulin degludec/liraglutide on glycated hemoglobin levels in patients with uncontrolled type 2 diabetes: The DUAL V randomized clinical trial. JAMA 315: 898-907, 2016.

2. Pfeffer MA, Claggett B, Diaz R, Dickstein K, Gerstein HC, Køber LV, Lawson FC, Ping L, Wei X, Lewis EF, et al: Lixisenatide in patients with type 2 diabetes and acute coronary syndrome. N Engl J Med 373: 2247-2257, 2015.
3. Bader M: ACE2, angiotensin-(1-7), and mas: The other side of the coin. Pflugers Arch 465: 79-85, 2013.

4. Hamming I, Cooper ME, Haagmans BL, Hooper NM, Korstanje R, Osterhaus AD, Timens W, Turner AJ, Navis G and van Goor H: The emerging role of ACE2 in physiology and disease. J Pathol 212: 1-11, 2007.

5. Zhang LH, Pang XF, Bai F, Wang NP, Shah AI, McKallip RJ, Li XW, Wang X and Zhao ZQ: Preservation of glucagon-like peptide-1 level attenuates angiotensin II-induced tissue fibrosis by altering AT1/AT 2 receptor expression and angiotensin-converting enzyme 2 activity in rat heart. Cardiovase Drugs Ther 29: 243-255, 2015.

6. Tikellis C, Pickering R, Tsorotes D, Du XJ, Kiriazis H, Nguyen-Huu TP, Head GA, Cooper ME and Thomas M: Interaction of diabetes and ACE2 in the pathogenesis of cardiovascular disease in experimental diabetes. Clin Sci (Lond) 123: 519-529, 2012.

7. Zygalaki E, Kaklamanis L, Nikolaou NI, Kyrzopoulos S Houri M, Kyriakides Z, Lianidou ES and Kremastinos DT: Expression profile of total VEGF, VEGF splice variants and VEGF receptors in the myocardium and arterial vasculature of diabetic and non-diabetic patients with coronary artery disease. Clin Biochem 41: 82-87, 2008.

8. Jesmin S, Zaedi S, Shimojo N, Iemitsu M, Masuzawa K, Yamaguchi N, Mowa CN, Maeda S, Hattori Y and Miyauchi T: Endothelin antagonism normalizes VEGF signaling and cardiac function in STZ-induced diabetic rat hearts. Am J Physiol Endocrinol Metab 292: E1030-E1040, 2007.

9. Chitose T, Sugiyama S, Sakamoto K, Shimomura H, Yamashita T, Hokamaki J, Tsunoda R, Shiraishi S, Yamashita Y and Ogawa H: Effect of a hydrophilic and a hydrophobic statin on cardiac salvage after ST-elevated acute myocardial infarction-a pilot study. Atherosclerosis 237: 251-258, 2014

10. Muhlestein JB, Lappé DL, Lima JA, Rosen BD, May HT, Knight S, Bluemke DA, Towner SR, Le V, Bair TL, et al: Effect of screening for coronary artery disease using CT angiography on mortality and cardiac events in high-risk patients with diabetes: The FACTOR-64 randomized clinical trial. JAMA 312: 2234-2243, 2014.

11. Dombi T, Kwok KK and Sultan MB: A retrospective, pooled data analysis of the safety of pegaptanib sodium in the treatment of age-related macular degeneration in subjects with or without diabetes mellitus. BMC Ophthalmol 12: 37, 2012.

12. Li W, Zhao W, Wu Q, Lu Y, Shi J and Chen X: Puerarin improves diabetic aorta injury by inhibiting NADPH oxidase-derived oxidative stress in STZ-induced diabetic rats. J Diabetes Res 2016: 8541520, 2016.

13. Shukla R, Pandey N, Banerjee S and Tripathi YB: Effect of extract of Pueraria tuberosa on expression of hypoxia inducible factor- $1 \alpha$ and vascular endothelial growth factor in kidney of diabetic rats. Biomed Pharmacother 93: 276-285, 2017.

14. Gao Y, Wang X and He C: An isoflavonoid-enriched extract from Pueraria lobata (kudzu) root protects human umbilical vein endothelial cells against oxidative stress induced apoptosis. J Ethnopharmacol 193: 524-530, 2016.

15. Ai F, Chen M, Yu B, Yang Y, Xu G, Gui F, Liu Z, Bai X and Chen Z: Puerarin accelerates cardiac angiogenesis and improves cardiac function of myocardial infarction by upregulating VEGFA, Ang-1 and Ang-2 in rats. Int J Clin Exp Med 8: 20821-20828, 2015.

16. Liang J, Yin K, Cao X, Han Z, Huang Q, Zhang L, Ma W, Ding F, Bi C, Feng D, et al: Attenuation of low ambient temperature-induced myocardial hypertrophy by atorvastatin via promoting Bcl-2 expression. Cell Physiol Biochem 41: 286-295, 2017.

17. Mingrone G, Panunzi S, De Gaetano A, Guidone C, Iaconelli A, Nanni G, Castagneto M, Bornstein S and Rubino F: Bariatric-metabolic surgery versus conventional medical treatment in obese patients with type 2 diabetes: 5 year follow-up of an open-label, single-centre, randomised controlled trial. Lancet 386: 964-973, 2015.

18. Derosa G, Mugellini A, Pesce RM, D'Angelo A and Maffioli P: Barnidipine compared to lercanidipine in addition to losartan on endothelial damage and oxidative stress parameters in patients with hypertension and type 2 diabetes mellitus. BMC Cardiovasc Disord 16: 66, 2016. 
19. Strand E, Pedersen ER, Svingen GF, Schartum-Hansen H, Rebnord EW, Bjørndal B, Seifert R, Bohov P, Meyer K, Hiltunen JK, et al: Dietary intake of n-3 long-chain polyunsaturated fatty acids and risk of myocardial infarction in coronary artery disease patients with or without diabetes mellitus: A prospective cohort study. BMC Med 11: 216, 2013.

20. McMullan CJ, Lambers Heerspink HJ, Parving $\mathrm{HH}$, Dwyer JP, Forman JP and de Zeeuw D: Visit-to-visit variability in blood pressure and kidney and cardiovascular outcomes in patients with type 2 diabetes and nephropathy: A post hoc analysis from the RENAAL study and the Irbesartan Diabetic Nephropathy Trial. Am J Kidney Dis 64 714-722, 2014.

21. Jakljević T, Ruzić A, Bazdarić K, Zaputović L, Mavrić Z, Champagne $S$ and Teiger E: Detection of myocardial ischemia in diabetic patients: The limitations of myocardial perfusion imaging. Coll Antropol 36: 821-826, 2012.

22. Drefs M, Thomas MN, Guba M, Angele MK, Werner J, Conrad M, Steib CJ, Holdt LM, Andrassy J, Khandoga A and Rentsch M: Modulation of glutathione hemostasis by inhibition of 12/15-lipoxygenase prevents ROS-mediated cell death after hepatic ischemia and reperfusion. Oxid Med Cell Longev 2017: 8325754, 2017.

23. Al-Taweel AM, Raish M, Perveen S, Fawzy GA, Ahmad A, Ansari MA, Mudassar S and Ganaie MA: Nepeta deflersiana attenuates isoproterenol-induced myocardial injuries in rats: Possible involvement of oxidative stress, apoptosis, inflammation through nuclear factor $(\mathrm{NF})-\mathrm{\kappa B}$ downregulation. Phytomedicine 34: 67-75, 2017.
24. Patel VB, Mori J, McLean BA, Basu R, Das SK, Ramprasath T, ParajuliN,Penninger JM, GrantMB,Lopaschuk GD and OuditGY: ACE2 deficiency worsens epicardial adipose tissue inflammation and cardiac dysfunction in response to diet-induced obesity. Diabetes 65: 85-95, 2016.

25. Iribarren C, Phelps BH, Darbinian JA, McCluskey ER, Quesenberry CP, Hytopoulos E, Vogelman JH and Orentreich N: Circulating angiopoietins-1 and -2, angiopoietin receptor Tie-2 and vascular endothelial growth factor-A as biomarkers of acute myocardial infarction: A prospective nested case-control study. BMC Cardiovasc Disord 11: 31, 2011.

26. Shams AS, Mohammed MH, Loka MM and Abdel Rahman GM: Assessment of the protective role of prenatal zinc versus insulin supplementation on fetal cardiac damage induced by maternal diabetes in rat using caspase-3 and KI67 immunohistochemical stains. Cardiol Res Pract 2016: 7469549, 2016.

27. Li H, Liu Z, Wang J, Wong GT, Cheung CW, Zhang L, Chen C, Xia Z and Irwin MG: Susceptibility to myocardial ischemia reperfusion injury at early stage of type 1 diabetes in rats. Cardiovasc Diabetol 12: 133, 2013.

28. Yan J, Duan J, Wu X, Guo C, Yin Y, Zhu Y, Hu T, Wei G, Wen A and Xi M: Total saponins from Aralia taibaiensis protect against myocardial ischemia/reperfusion injury through AMPK pathway. Int J Mol Med 36: 1538-1546, 2015.

29. Chu LM, Osipov RM, Robich MP, Feng J, Sheller MR and Sellke FW: Effect of thrombin fragment (TP508) on myocardial ischemia reperfusion injury in a model of type 1 diabetes mellitus. Circulation 122 (11 Suppl): S162-S169, 2010. 